\title{
ODEs and Redefining the Concept of Elementary Functions
}

\author{
Alexander Gofen \\ The Smith-Kettlewell Eye Research Institute, 2318 Fillmore St., San Francisco, CA 94102, \\ USA \\ galex@ski.org，www.ski.org/gofen
}

\begin{abstract}
The modern concept of elementary functions and the Taylor method are deeply connected. The article summarizes the remarkable (although not widely known) facts about the modern Taylor method, Ordinary Differential Equations (ODEs) and the modern notion of elementary functions - the property that actually takes place for all ODEs used in applications. Beside the typical usage of the Taylor method for integrating initial value problems, two new applications of the Taylor method are considered: integrating until the given end value of a dependent variable, and integrating the boundary value problem.
\end{abstract}

In the bright memory of the late Prof. Michael L. Lidov

\section{Introduction}

According to the tradition since the 19th century, elementary functions were defined just by a convention as a certain limited list of well studied functions, a desirable final format to express the solutions of different problems. They were polynomials and rational functions (of several variables), plus the exponential, logarithmic, trigonometric and the inverse trigonometric functions (of one variable), plus all finite superpositions of them (The Mathematical Encyclopedia).

Liouville adhered to a slightly different definition: he considered elementary also algebraic functions, integrals, exponential of integrals, solutions of linear differential equations with constant coefficients, and all functions which can be obtained by repeated iteration of these functions.

In any case, it was just a rather artificial convention. Probably it was Ramon Moore [1], who first suggested another approach to define the elementary functions based not on an arbitrary convention, but on a quite fundamental property, that the elementary functions are solutions of ODEs with rational right hand parts. Although his goal was mostly to improve and optimize the algorithm of the classical Taylor method such a way that the modern Taylor method would require no more than $O\left(N^{2}\right)$ operations, the new approach appeared to have also a profound mathematical meaning and connections. It established a fundamental dividing line between non-elementary functions and the elementary ones. The latter characterize the types of ODEs for which the modern Taylor method is applicable, and they also provide a constructive

P.M.A. Sloot et al. (Eds.): ICCS 2002, LNCS 2329, pp. 1000-1009, 2002.

(C) Springer-Verlag Berlin Heidelberg 2002 
way how (almost) any (!) non-linear source system of ODEs may be transformed to quite remarkable forms - even as simple as quadratic polynomials (Table 2).

First of all, the modern Taylor method was intended as a powerful numeric method to integrate the initial value problems with the ultimate accuracy. The software implementing it was known since the 80s, and the recent version of such an integrated environment for PCs (The Taylor Center) is described in these proceedings [6,7].

This paper considers also a couple of less typical tasks for the Taylor method: integration until the given end value of the dependent variable, and the boundary value problem.

\section{Elementary Functions and ODEs}

This section summarizes the fundamental facts about the modern elementary functions - the basis of the modern Taylor method.

Definition 1: a (vector-) function $f=\left\{f_{k}\left(x_{1}, \ldots, x_{m}\right)\right\},(k=1, \ldots, n)$, is called elementary with respect to $x_{1}$ if there exists a system of $N \geq n$ ODEs

$$
\partial f_{k} / \partial x_{1}=R_{k l}\left(f_{1}, \ldots, f_{N}\right), \quad k=1, \ldots, N
$$

with the rational right hand parts $R_{k l}$, for which $f$ is a solution ( $f$ is defined by this system as a function of $x_{1}$ ). We say that the (vector-) function $f$ is elementary if it is elementary by all its variables $x_{1}, \ldots, x_{m}$, thus there exist $m$ different systems of ODEs $(l=1, \ldots m)$ defining $f$ as a (vector-) function of each of its arguments $x_{l}$. All those rational right hand parts $R_{k l}$ may be organized into a matrix, called the matrix of elementarity of the (vector-) function $f$.

It was proved [2], that if we consider a similar Definition 1' (a version of Definition 1), with the polynomial right hand parts $P_{k l}$ rather than the rational $R_{k l}$, both definition are equivalent.

In order to demonstrate elementarity of a (vector-) function, it may appear necessary to consider it as a component of a larger vector-function. For example, $f(x, y, z)=$ $\cos (x) e^{y} \zeta(z)$ is elementary together with $g(x, y, z)=\sin (x) e^{y} \zeta(z)$ by $x,\left(f_{x}^{\prime}=-g ; g_{x}{ }^{\prime}=f\right)$, it is elementary "itself" by $y\left(f_{y}^{\prime}=f\right)$, and it is probably not elementary by $z$ (Zeta function of Riemann). Thus, the matrix of elementarity by $x, y$ for function $f$ (as a component of the vector-function $\{f, g\})$ is this:

$$
\left[\begin{array}{ll}
-g & f \\
f & 0
\end{array}\right]
$$

The function $w=\tan (t)$ is elementary together with $u=\cos (t)$ and $v=\sin (t)$, and the matrix of elementarity is 


$$
\left(\begin{array}{lll}
-v & u & 1 / u^{2}
\end{array}\right)^{\mathrm{T}}
$$

Table 1. Theorems and facts about the elementary functions

\begin{tabular}{|c|c|c|}
\hline 1 & Polynomial and rational functions & Elementary \\
\hline 2 & $\begin{array}{l}\text { All traditionally elementary functions, as well as those } \\
\text { by Liouville }\end{array}$ & Elementary \\
\hline 3 & Superposition of elementary vector-functions & Elementary \\
\hline 4 & Inverse to an elementary vector-function & Elementary \\
\hline 5 & $\begin{array}{l}\text { Implicit functions } x_{k} \text { of an equation } F\left(x_{1}, x_{2}, \ldots x_{n}\right)=0 \text { with } \\
\text { an elementary function } F\end{array}$ & Elementary \\
\hline 6 & Algebraic functions & Elementary \\
\hline 7 & Derivative of an elementary function & Elementary \\
\hline 8 & $\begin{array}{l}\text { Indefinite integral with respect to a certain variable } \\
\text { (say } t \text { ) of an elementary function } f(t, x)\end{array}$ & $\begin{array}{l}\text { Elementary by } t \text {, but } \\
\text { not necessarily by } x\end{array}$ \\
\hline 9 & $\begin{array}{l}\text { Vector-function } u_{k}(t, x) \text { which is a solution of ODEs } \\
u_{k}^{\prime}=f_{k}\left(u_{1}, \ldots u_{n}, x\right) \text { with an elementary vector-function }\left\{f_{k}\right\}\end{array}$ & $\begin{array}{l}\text { Elementary by } t \text {, but } \\
\text { not necessarily by } x\end{array}$ \\
\hline 10 & $\lim f_{n}(t)$ with all functions $f_{n}(t)$ elementary & $\begin{array}{l}\text { Not necessarily } \\
\text { elementary }\end{array}$ \\
\hline 11 & $\sum_{n=0}^{\infty} a_{n} t^{n} \quad$ converging to $f(t)$. Coefficients $a_{n}$ are arbitrary & $\begin{array}{l}\text { Not necessarily } \\
\text { elementary }\end{array}$ \\
\hline 12 & $\begin{array}{l}\sum_{n=0}^{\infty} a_{n} t^{n} \quad \text { converging to } f(t) \text {. Coefficients } a_{n} \text { are obtained } \\
\text { via the special recurrent formulas for differentiating the } \\
\text { canonical equations }\end{array}$ & Elementary \\
\hline 13 & $\begin{array}{l}\text { A solution } x(t) \text { of a finite difference equation } \\
F(x(t), x(t+h))=0 \text { with an elementary non-linear } \\
\text { function } F\end{array}$ & $\begin{array}{l}\text { Not necessarily } \\
\text { elementary }\end{array}$ \\
\hline 14 & Euler's Gamma function defined as $\Gamma(x+1)=x \Gamma(x)$ & Non-elementary \\
\hline
\end{tabular}

An algebraic function $x=X(y, z)$, defined by an implicit equations $P(x, y, z)=0$, where $P$ is a polynomial of arbitrary high degree, is elementary by $y$ and $z$ :

$$
X_{y}^{\prime}=-P_{y}^{\prime}(X, y, z) / P_{x}^{\prime}(X, y, z) ; \quad X_{z}^{\prime}=-P_{z}^{\prime}(X, y, z) / P_{x}^{\prime}(X, y, z) \text {. }
$$


This demonstrates existence of elementary functions of more than one variable, which are not rational (a case not covered by the definition of Moore [1]). Also, each of these ODEs gives an example, when the solution $X(y, z)$ is elementary both by its parameter and the differential variable, which is not always the case, but rather an exception.

The fundamental properties of elementary functions are summarized in Table 1. This modern definition of elementarity includes all functions, which traditionally belong to that notion, plus all solutions of ODEs with the rational right hand parts, all their possible superpositions and inverse (vector-) functions. Well, then, do non-elementary functions exist at all according to the modern definition? It is not so easy to point out even one. All types of functions in Table 1 mentioned as "not necessarily elementary" are rather "good candidates". Meanwhile the non-elementarity is known for sure only for Euler's Gamma function $\Gamma(x)$ (due to the theorem of Hödel, stating that $\Gamma(x)$ cannot be a solution of any polynomial ODE).

Table 2. The remarkable transformations possible for all ODEs with the right hand parts being elementary vector-function (practically any system of ODEs used in applications)

A standard system of ODEs whose right hand parts are an elementary vector-function (practically any system of ODEs used in applications) can be reduced to...

A system of ODEs whose right hand parts are the rational functions [2]. It can be further reduced to...

A canonical system, a mixture of the algebraic and differential equations of certain simple types, used to compute efficiently the $\mathrm{N}$-order derivatives with no more than $O\left(N^{2}\right)$ operations.
A system of ODEs whose right hand parts are the polynomial functions [2]. It can be further reduced to...

A system of ODEs whose right hand parts are the polynomials of a degree $\leq \mathbf{2}$ (see [4]). It can be further reduced to...

A system of ODEs whose right A system of ODEs hand parts are the polynomials whose right hand parts of a degree 2 with coefficients are the polynomials $-\mathbf{1 , 0}$ or $\mathbf{1}$ only (see [5]). with square terms only (see [4]).

Is there any sense in defining so large a concept of the elementary functions that practically all functions used in applications belong to this class? The great reason is that for all ODEs with elementary right hand parts there exists a unified, constructive and efficient way of transforming them to the rational and several other remarkable forms, including the canonical, which makes possible the efficient modern Taylor method. These transformations are summarized in Table 2.

Each transformation (from the upper to lower cells in this Table) results in a certain increase in the number of equations. But the transformation required for the modern Taylor method 


\section{Any Elementary ODEs $\rightarrow$ Rational ODEs $\rightarrow$ Canonic System}

adds just as little as is necessary to define the non-rational elementary functions [2]. On the contrary, transforming to the polynomial formats of a degree $\leq 2$ may multiply the number of equations essentially $[4,5]$. The fact itself that it is possible seems remarkable in any case.

Even with the notion of elementarity that large, it does impose certain applicability limits for the Taylor method. For example, we must be aware that the solutions of ODEs as functions of the parameters mostly are not elementary, thus we cannot apply the Taylor method to further integrate them by these parameters. Similarly, double and multiple definite integrals also cannot be computed with the Taylor method directly. On the other hand, if we know a (first, a second) integral of a certain system of ODEs, it is usually an implicit equation built of elementary functions of unknown variables and parameters. By these parameters, elementarity of the solution does take place.

\section{Modern Taylor Method Basics}

First, we assume a convention. In Modern Taylor Method, the concept of $n$-th derivative means the so called normalized derivative, which by definition is

$$
u^{[n]}=u^{(n)} / n !
$$

so that the Taylor expansion looks like this:

$$
u(t)=\sum_{u^{[k]}\left(t-t_{0}\right)^{k}}
$$

(further on, the conventional notation $u^{(k)}$ is used, although we always mean the normalized derivatives).

What distinguishes the modern Taylor method from its classical counterpart is the special technique of computing derivatives. Let us consider a standard initial value problem for a system of ODEs with any analytical right hand parts

$$
u_{k}^{\prime}=f_{k}\left(u_{1}, \ldots, u_{m}\right),\left.\quad u_{k}\right|_{t=a}=a_{k}, \quad k=1, \ldots m
$$

With no additional assumptions about the right hand parts (2), to perform N-order differentiation, we have nothing simpler than the formula of Faa di-Bruno [2] for $\mathrm{N}$-order derivatives of a superposition of functions - quite a complex formula with exponential growth, completely impractical.

Even for a more specific right hand part, for example like $x^{\prime}=u v w y$, the Leibnitz formula for several factors is needed 


$$
(u v w y)^{(N)}=\sum \sum \sum u^{(i)} v^{(j)} w^{(k)} y^{(N-i-j-k)}
$$

That requires as many as $O\left(N^{4}\right)$ operations in this specific case. What is the key issue for the modern Taylor method is a possibility to reduce whichever complex (but elementary!) right hand parts to a sequence of simple canonical equations [2,3], or to a sequence of formulas over two operands, for which computing $\mathrm{N}$-order derivatives never requires more than $O\left(N^{2}\right)$ operations. Such formulas are for example, the Leibnitz formula for $\mathrm{N}$-th derivative of a product of $t w o$ functions:

$$
(u v)^{(N)}=\sum_{i=0}^{N} u^{(i)} v^{(N-i)}
$$

The similar by complexity $O\left(N^{2}\right)$ formulas exist for quotient, power function, exponent and logarithm, for example

$$
\begin{gathered}
\text { if } R=u / v \text {, then } R^{(N)}=\left(u^{(N)}-\sum_{i=0}^{N-1} R^{(i)} u^{(N-i)}\right) / v \text {; } \\
\text { if } R=u^{a} \text {, then } R^{(N)}=\left(\sum_{i=0}^{N-1}(a(1-i / N)-i / N) R^{(i)} u^{(N-i)}\right) / u, \quad(a \text { is a constant }) .
\end{gathered}
$$

For the linear equations like $a u, u+v$ the corresponding formulas are trivial and require $O(n)$ operations only.

Note: the canonic equations or the sequence of formulas must be computed in the given order only (not a good case for parallel computing), but the polynomial forms of a degree $\leq 2$ may be used instead of the canonical equations, and unlike the latter, they may be computed in any order concurrently.

Thus, the modern Taylor method allows obtaining the numeric values of N-order derivatives in any given point for the system (2) and then summing the Taylor expansion. As soon as the source system (2) is reduced to the rational right hand parts (possibly allowing also the power, exponent and logarithm), an algorithm, similar to parsing an arithmetic expression, automatically performs further reducing to certain internal structures (like the implicit canonic system), and computes the Taylor expansion. That way the Taylor method integrates the initial value problems, applying finite steps not approaching zero and still maintaining any required high accuracy up to the ultimate - all available digits of the given binary representation $[6,7]$. Practically, it is reasonable to select the order $\mathrm{N}$ of the Taylor method according to Moore's "rule of thumb" [1-3] - a value between 20 and 30. The heuristic radius of convergence may be obtained from the series of the derivatives $[6,7]$. 


\section{Reaching the Given End Value}

The initial value problem is a "native" one for the Taylor method, but in an application it may be necessary to integrate until the moment when the given end value of the dependent variable is reached: that would allow determining a period of the solution (if it exists). Specifically, we are going to integrate say $u_{l}(t)$ until it reaches a certain value $b_{l}$. To do that, there are two approaches in the framework of the Taylor method: (1) It's always possible and easy to write the systems of ODEs for the inverse function $T\left(u_{l}\right)$ and to integrate it by now independent variable $u_{l}$ until $b_{l}$; (2) To apply the standard technique of numeric analysis of polynomials to determine whether there is a root of $u_{l}(t)=P_{I}(t)=b_{l}$ in the current domain of the Taylor expansion for $u_{l}(t)$.

\subsection{Integrating the Inverse Function}

Given the source system (2), the ODEs for the function $T\left(u_{1}\right)$ inverse to $u_{l}(t)$ are

$$
\begin{aligned}
& \partial u_{k} / \partial u_{l}=f_{k}\left(u_{l}, \ldots, u_{m}\right) / f_{l}\left(u_{l}, \ldots, u_{m}\right), \quad k=1, \ldots m \\
& \partial T / \partial u_{l}=1 / f_{l}\left(u_{l}, \ldots, u_{m}\right)
\end{aligned}
$$

True, when switching to system (4) with the independent variable $u_{l}$, we must be ready to run into singularities while integrating from a current point to the end value $b_{l}$. We may have to edge into the complex plane temporarily to circumvent the singularities, and to take into consideration different Riemann planes. Or we can just switch back to the system (2) and integrate it by $t$ while near the singularity, and then return back to integration by $u_{l}$ again.

\subsection{Analyzing the Polynomial}

At each step of integration by $t$, we may obtain a polynomial expansion $P_{l}(t)$, representing the solution $u_{l}(t)$ with the ultimate accuracy in a certain finite domain. Thus, without sacrificing any accuracy, we can explore the equation $P_{l}(t)=b_{l}$ in the domain applying the well developed technique for polynomials. That would make possible either to conclude that there are no solutions (and to perform the next step of integration by $t$ ), or to obtain the solution $t_{1}$ delivering the end condition $u_{1}\left(t_{1}\right)=b_{1}$.

\section{Boundary Value Problems}

Lets us consider a boundary value problem

$$
\begin{gathered}
u_{k}^{\prime}=f_{k}\left(u_{1}, \ldots, u_{m}\right), \quad k=1, \ldots m ;\left.\quad u_{k}\right|_{t=0}=a_{k}, \quad k=2, \ldots m \\
\left.u_{1}\right|_{t=0}=x,\left.\quad u_{1}\right|_{t=1}=b_{1}
\end{gathered}
$$


with the unknown component $x$ of the initial value. Given the incomplete initial condition $u_{1}=x$ at $t=0$, the unknown solution $u_{k}(t, x)$ in the general case is not elementary by $x$, therefore there is no hope of finding a system for the inverse function $X\left(t, u_{l}\right)$ and following the approach 4.1. Instead, to obtain the N-order Taylor expansion of the solution $u$ by $x$ at the end point $t=1$, we can adjust the approach 4.2. That will require $\mathrm{N}$-times expanding the source system (5), adding ODEs defining all $\partial^{n} u_{k} / \partial x^{n}$ as functions of $t$. (Practically, the order $\mathrm{N}$ of the Taylor method is usually 20$30)$.

Here is how it is done.

$u_{k}^{\prime}=f_{k}\left(u_{l}, \ldots, u_{m}\right),\left.\quad u_{1}\right|_{t=0}=x,\left.\quad u_{k}\right|_{t=0}=a_{k}, k=2, \ldots m$, (the source ODEs).

Then, we may add:

$$
\begin{array}{ll}
\partial u_{k}^{\prime} / \partial x=\partial f_{k} / \partial x, & \partial u_{t} /\left.\partial x\right|_{t=0}=1, \quad \partial u_{k} /\left.\partial x\right|_{t=0}=0, \quad k=2, \ldots m ; \\
\partial^{2} u_{k}^{\prime} / \partial x^{2}=\partial^{2} f_{k} / \partial x^{2}, & \partial^{2} u_{k} /\left.\partial x^{2}\right|_{t=0}=0, \quad k=1, \ldots m ; \\
\partial^{N} u_{k}^{\prime} / \partial x^{N}=\partial^{N} f_{k} / \partial x^{N}, & \partial^{N} u_{k} /\left.\partial x^{N}\right|_{t=0}=0, \quad k=1, \ldots m ;
\end{array}
$$

Now, if we start with an arbitrary parameter $x$, after integrating this system from $t=0$ until $t=1$, we will obtain the sequence in this point

$$
\partial u_{l} / \partial x, \partial^{2} u_{l} / \partial x^{2}, \ldots . ., \partial^{N} u_{l} / \partial x^{N}
$$

which makes available the Taylor expansion by $x$ of $u_{l}(t, x)$ at $t=1$ (as though $u_{l}(t, x)$ were elementary by $x$ and we had the corresponding system of ODEs):

$$
u_{l}(t, x+\Delta x)=u_{l}(t, x)+\left(\partial u_{l} / \partial x\right) \Delta x+\left(\partial^{2} u_{l} / \partial x^{2}\right) \Delta x^{2}+\ldots+\left(\partial^{N} u_{l} / \partial x^{N}\right) \Delta x^{N}
$$

(the derivatives are normalized). Now, similarly to what we do with respect to $t$, we can estimate the domain of convergence by $x$ of this Taylor expansion, and then analyze if there is a root of the following polynomial of $\Delta x$

$$
u_{l}(t, x)+\left(\partial u_{l} / \partial x\right) \Delta x+\left(\partial^{2} u_{l} / \partial x^{2}\right) \Delta x^{2}+\ldots+\left(\partial^{n} u_{l} / \partial x^{n}\right) \Delta x^{n}=b_{l} .
$$

Here we are in a position similar to that in section 4.2 above (except that it took much more efforts to obtain this expansion). If there is no solution in the domain, we increment $x+\Delta x$ and repeat this whole step.

A question may arise how we would obtain all these right hand parts $\partial^{N} f_{k} / \partial x^{N}$ of those additional $\mathrm{Nm}$ equations (6). Surprising as it is, we should not care at all: the right hand parts of the source equations (5) do not depend explicitly on $x$ : instead, the parameter $x$ occurs in the initial values. Therefore, the same algorithm (the same 
postfix sequence and the instructions encoding it) [6-8], performing the automatic differentiation by $t$, would do that also by $x$, writing the corresponding results into the array (with one dimension more). It would perform it in the embedded loops: external loop by $t$-differentiation, the internal - by $x$-differentiation. (This algorithm will be added to the existing version of the Taylor Center computer program [6-8] in the future).

Thus, the automatic differentiation by the parameter $x$ appeared to be also possible even without the assumption about the elementarity by $x$ of the solution. If this elementarity really took place and we knew the ODEs defining $u_{l}(t, x)$ with respect to $x$, we would be able to integrate them by $x$ just once in the end point $t=1$. Otherwise, we have to do that at each $t$-step (integrating $\mathrm{N}$ times larger system). In other words, in order to have available both $\partial^{n} u_{k} / \partial t^{n}$ and $\partial^{n} u_{k} / \partial x^{n}, n=1,2, \ldots, N$, we compute $N^{2}$ derivatives of the source system (5) at each step (vs. just $N$ derivatives for the initial value problem by $t$ ).

Note: the same approach remains valid also for integrating the source system depending on two or more parameters as soon as the parameters are in the initial values (rather than in the right hand parts). For example, for two parameters $x, y$, (plus $t$ ) the number of computed derivatives would be $2 N^{2}$, i.e. it grows linearly with number of the parameters (unlike the number of the mixed partial derivatives).

Another concern indeed is that the $x$-convergence radius at the $e n d$ point $t=1$ may be extremely small (depending on the instability of the problem) so that a lot of $x$-integration steps would be needed in order to reach the given value $b_{1}$.

\section{Conclusions}

The modern concept of elementary functions reveals the deep connections between seemingly unrelated issues such as the elementarity vs. :

(1) Existence of formulas for obtaining N-order derivatives with efficiency $O\left(N^{2}\right)$;

(2) Reducing of any arbitrary non-linear ODEs to surprisingly simple forms.

It also shows fundamental differences in:

(3) The nature of the dependency on $t$ of the solutions of ODEs vs. the dependency on the parameters;

(4) The nature of the solutions of non-linear finite difference equations vs. those of ODEs.

The automatic differentiation by parameters of the solution of ODEs could be just as simple as switching to another system of ODEs with no increase in the per-step computation, if the dependency on the parameters of the solution were also elementary and the corresponding systems were known. Even with no elementarity by 
the parameters, the automatic differentiation by them is still possible, providing they are placed in the initial conditions. Then the volume of computation at each step grows proportionally with the number of parameters.

The modern Taylor method is an efficient integrator with unique features especially important when the ultimate accuracy is the main goal. Typically used for initial value problems, it may be adjusted also for boundary value problems or integration until the given end value of the unknown function. The integration by a parameter is to be added in the future to the software package for PCs [6-8], which introduces an integrating environment for exploring and graphing solutions of ODEs implemented with all the features of the Graphical User Interface.

Acknowledgement: I am very thankful to Dr. Dmitriy Sonechkin, who first pointed me out and provided the references to the remarkable transformations [4, 5] for ODEs, when I enjoyed working together with him in the Hydrometeorological Center (Moscow, Russia) in 80s.

\section{References}

1. Moore, R. E.: Interval Analysis. Prenitce-Hall, Englewood Cliffs, N.Y. (1966).

2. Gofen, A. M.: Fast Taylor-Series Expansion and the Solution of the Cauchy Problem. U.S.S.R. Comput. Maths. Math. Phys. Vol. 22, No. 5, pp. 74-88 (1982)

3. Gofen, A. M.: Taylor Method of Integrating Ordinary Differential Equations: the Problem of Steps and Singularities. Cosmic Research, Vol. 30, No. 6, pp. 581-593 (1992)

4. Charnyi, V. I.: Two Methods of integrating the equations of motion. Cosmic Research, Vol. 8, No. 5 (1970)

5. Kerner, E. H.: Universal Formats for Nonlinear Differential Systems. J. Math. Phys. Vol. 22, No. 7 (1981)

6. Gofen, A. M.: Recursive Journey to Three Bodies. Delphi Informant Magazine. Vol. 8, No. 3 (2002)

7. Gofen, A. M.: The Taylor Center for PCs: Graphing and Integrating ODEs with the Ultimate Accuracy. These Proceedings (2002)

8. Gofen, A. M.: The Taylor Center Demo for PCs.

http://www.ski.org/rehab/mackeben/gofen/TaylorMethod.htm 